\title{
Posterolateral percutaneous endoscopic discectomy with free-running electromyography monitoring under general anesthesia
}

\author{
Yoshihiro Kitahama', Genichiro Matsui ${ }^{2}$, Manabu Minami', Taigo Kawaoka', Kimimichi Otome², Masato Nakamura ${ }^{2}$ \\ ${ }^{1}$ Center for Spinal Surgery, Omaezaki Municipal Hospital, Omaezaki, Shizuoka 437-1696, Japan. \\ ${ }^{2}$ Department of Anesthesiology, Omaezaki Municipal Hospital, Omaezaki, Shizuoka 437-1696, Japan.
}

Correspondence to: Dr. Yoshihiro Kitahama, Center for Spinal Surgery, Omaezaki Municipal Hospital, 2060 Ikeshinden, Omaezaki, Shizuoka 437-1696, Japan. E-mail: ykitah@gmail.com

How to cite this article: Kitahama Y, Matsui G, Minami M, Kawaoka T, Otome K, Nakamura M. Posterolateral percutaneous endoscopic discectomy with free-running electromyography monitoring under general anesthesia. Mini-invasive Surg 2017;1:109-14.

\author{
Article history: \\ Received: 13 Mar 2017 \\ Accepted: 17 Jul 2017 \\ Published: 30 Sep 2017 \\ Key words: \\ Percutaneous endoscopic lumbar \\ discectomy, \\ electromyography, \\ lumbar disc herniation, \\ posterolateral approach, \\ numeric rating scale, \\ MacNab's criteria
}

\begin{abstract}
Aim: Posterolateral percutaneous endoscopic discectomy (PLPED) is commonly performed under local anesthesia, but patients and surgeons are concerned about intraoperative uncontrolled pain. The purpose of this study was to evaluate the safety of the PLPED under general anesthesia with free-running electromyography (EMG) monitoring. Methods: The clinical outcomes of consecutive 48 cases of lumbar disc herniation (LDH) were evaluated by numeric rating scale (NRS) score and MacNab's criteria. Hospital stay and time to ambulation and return to work were also assessed. Results: NRS score for the affected leg significantly improved from 6.4 to 0.9 immediately after the operation. MacNab's criteria were $91.5 \%$ for a follow-up period of 13.5 months. Although no serious complication occurred, 3 patients (6.3\%) had transient paresis that completely disappeared by 3 months. No recurrences were observed during the follow-up period. Conclusion: PLPED combined with EMG monitoring under general anesthesia is a safe and efficacious procedure for the treatment of LDH.
\end{abstract}

\section{INTRODUCTION}

Local anesthesia permits the performance of the posterolateral approach for percutaneous endoscopic discectomy (PLPED). ${ }^{[1]}$ In some cases, deep sedation is required for uncontrolled intraoperative pain. ${ }^{[2]}$ Uncontrolled intraoperative could lead to the early termination of the operation.

Local anesthesia is helpful to avoid exiting nerve root (ENR) injury from the patient's complaints during operative manipulation. ${ }^{[3]}$ Even under general anesthesia, free-running electromyography (EMG) monitoring succeeds in preserving the lumbar plexus for the extreme lateral approach. ${ }^{[4-7]}$

The purpose of this study was to evaluate the safety of PLPED under general anesthesia with EMG monitoring.

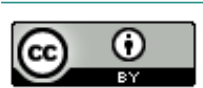

This is an open access article licensed under the terms of Creative Commons Attribution 4.0 International License (https://creativecommons.org/licenses/by/4.0/), which permits unrestricted use, distribution, and reproduction in any medium, as long as the original author is credited and the new creations are licensed under the identical terms.

For reprints contact: service@oaepublish.com

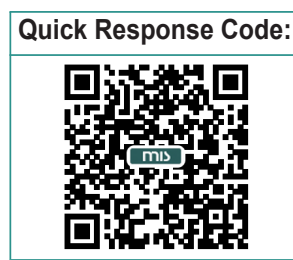




\section{METHODS}

\section{Ethics and patient consent}

This study was approved by the Ethics committee of the Omaezaki Municipal Hospital and all involved patients gave consent.

\section{Anesthesia and EMG monitoring}

Propofol and remifentanil hydrochloride-based general anesthesia was performed and muscle relaxants were only used at the initial stage for intubation. For free-running EMG monitoring, the needle electrodes were placed on 5 muscles (bilateral gluteus medius, hamstrings, quadriceps, tibialis anterior muscle, and gastrocnemius react) and the EMG was continuously recorded by Neurovision ${ }^{\circledast}$ free-running EMG monitoring system (NuVasive Inc., San Diego, CA, USA) [Figure 1].

\section{Preoperative measurement}

The direction and position of needle puncture were preoperatively designed by plain roentgenography (4 dimensional and 2 functional views), magnetic resonance imaging and computed tomography (CT) scan. Prone positioned CT scan enabled reproducibility and measured the actual operative situation. Axial CT image scanned paralleled with intervertebral disc provided information regarding entry points of the skin $(\mathrm{P})$ and the annulus fibrosus $(\mathrm{O})$ [Figure $2 \mathrm{~A}$ and $\mathrm{B}]$. A right angled triangle ( $\mathrm{P}-\mathrm{O}$ line is the hypotenuse) was made and designated as the intersection of the base and midline as I [Figure 2B]. The distances of P-I(x) were then calculated. Cranial $(\mathrm{y})$ and dorsal $(\mathrm{z})$ deviations from entry point of the annulus fibrosus $(\mathrm{O})$ were also calculated from CT and plain roentgenography (both anteroposterior and lateral views) [Figure 2B and C]. The calculated points on were drawn on patient's skin along with the anatomical landmarks (vertebral body, spinous process, transverse process, and iliac crest) to avoid incorrect puncture [Figure 3].

\section{Basic operative procedure}

The patients were carefully log rolled into the prone position. During the operation, a fluoroscope was placed across the center of the operating table to ensure appropriate positioning. An 8-mm skin and fascia incision were simultaneously made on calculated entry points of the skin $(P)$, and then an 18-gauge spinal needle was inserted into the annulus fibrosus. Epidurography with 1-2 $\mathrm{mL}$ of a contrast medium was first performed and then discography with $1.5 \mathrm{~mL}$ of liquid mixture (contrast medium:lidocaine:indigo carmine $=2: 2: 1$ ) was performed using the same needle. Following insertion of an obturator, a 7-mm diameter working sheath was inserted. Then, an endoscope [two different systems were used in this study: VERTEBRIS lumbar-thoracic ${ }^{\circledR}$ (Richard Wolf $\mathrm{GmbH}$, Knittlingen, Germany) and Spine TIP ${ }^{\circledR}$ (KARL STORZ GmbH, Tuttlingen, Germany)] was inserted and the lateral edge of the posterior longitudinal ligament (PLL) was confirmed in the center of the endoscopic operative field [Figure 4A].

Under endoscopic discectomy, herniated fragments were disconnected from the nucleus pulposus under the PLL. From the viewpoint of the PLL, preservation and consequently protection of the cauda equine, this procedure is important and an advantageous

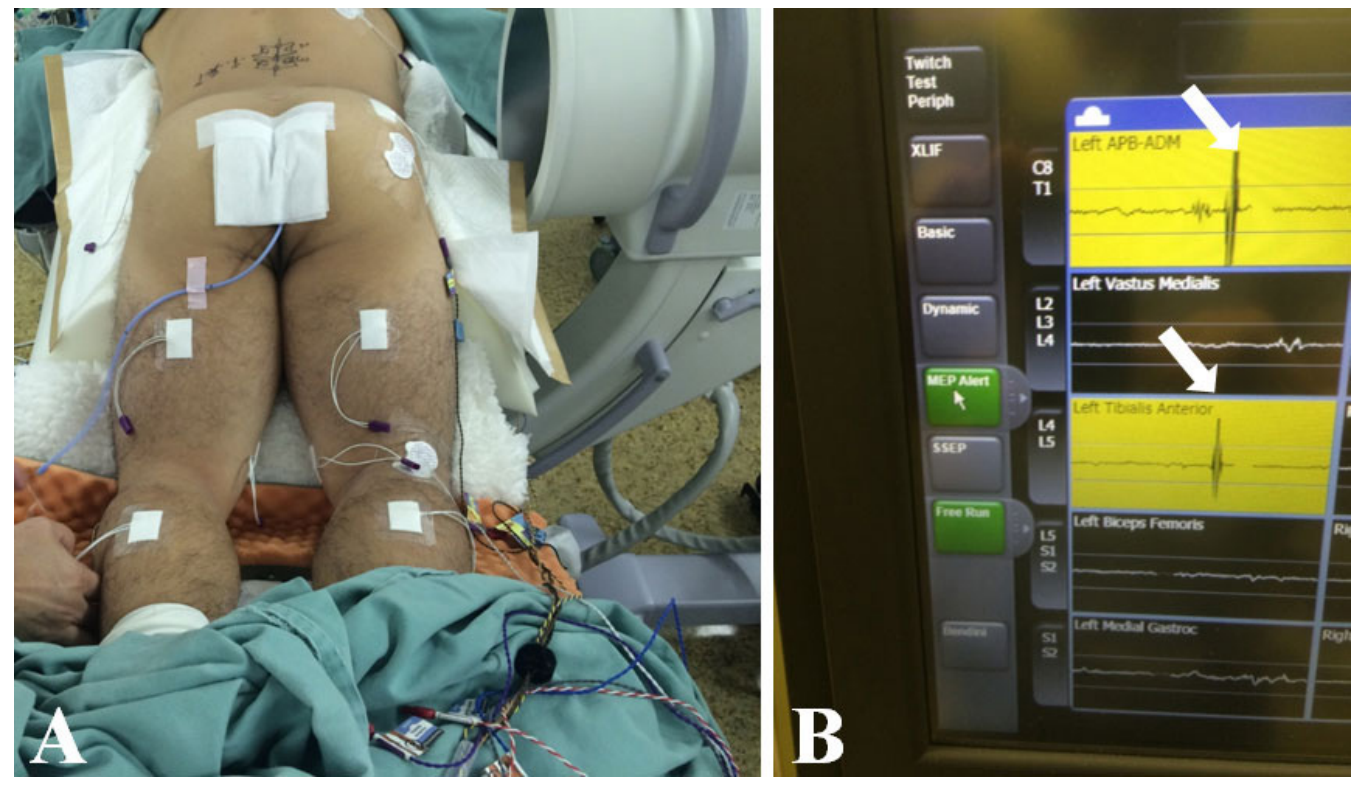

Figure 1: Intraoperative view of free-running electromyography (EMG) monitoring. (A)The needle electrodes are located on bilateral gluteus medius, hamstrings, quadriceps, tibialis anterior, and gastrocnemius; (B) exact waves of EMG from gluteus medius (upper) and tibialis anterior muscle (lower) are shown. Note that the waves reach the level of warning alarm (white arrows) 
operative step. To make the tunnels under the PLL, it is necessary to repeat 2-3 times of hand-down technique. ${ }^{[8]}$ When a piece of the fragment becomes visible from hiatus of the annulus fibrosus, it is possible to remove the fragment by an endoscope inserted from a horizontal direction. Although bleeding can cloud the view during discectomy and fragmentectomy, pin point and short time (within $6 \mathrm{~s}$ ) bipolar coagulation using a bipolar radio-frequency electrode system (Elliquence, Baldwin, NY, USA) allows hemostasis to be achieved. Microbubbles during operative manipulation can also cloud the view. Upward and downward motion of the endoscope in the outer sheath removes the bubbles and provides clear visualization. After decompression, the working sheath was carefully removed, and skin was closed with a single suture.

\section{Optional advanced procedures}

Partial facetectomy ${ }^{[9,10]}$ and pediculotomy ${ }^{[11]}$ were performed for total removal of the foraminal type LDH. For these techniques, an electrical high speed drill Primado $2^{\circledR}$ with Super Slim Attachment $200^{\circledR}$ (NSKNakanishi medical, Tochigi, Japan) was utilized. For the medial type of LDH, beak forceps were used for sharp
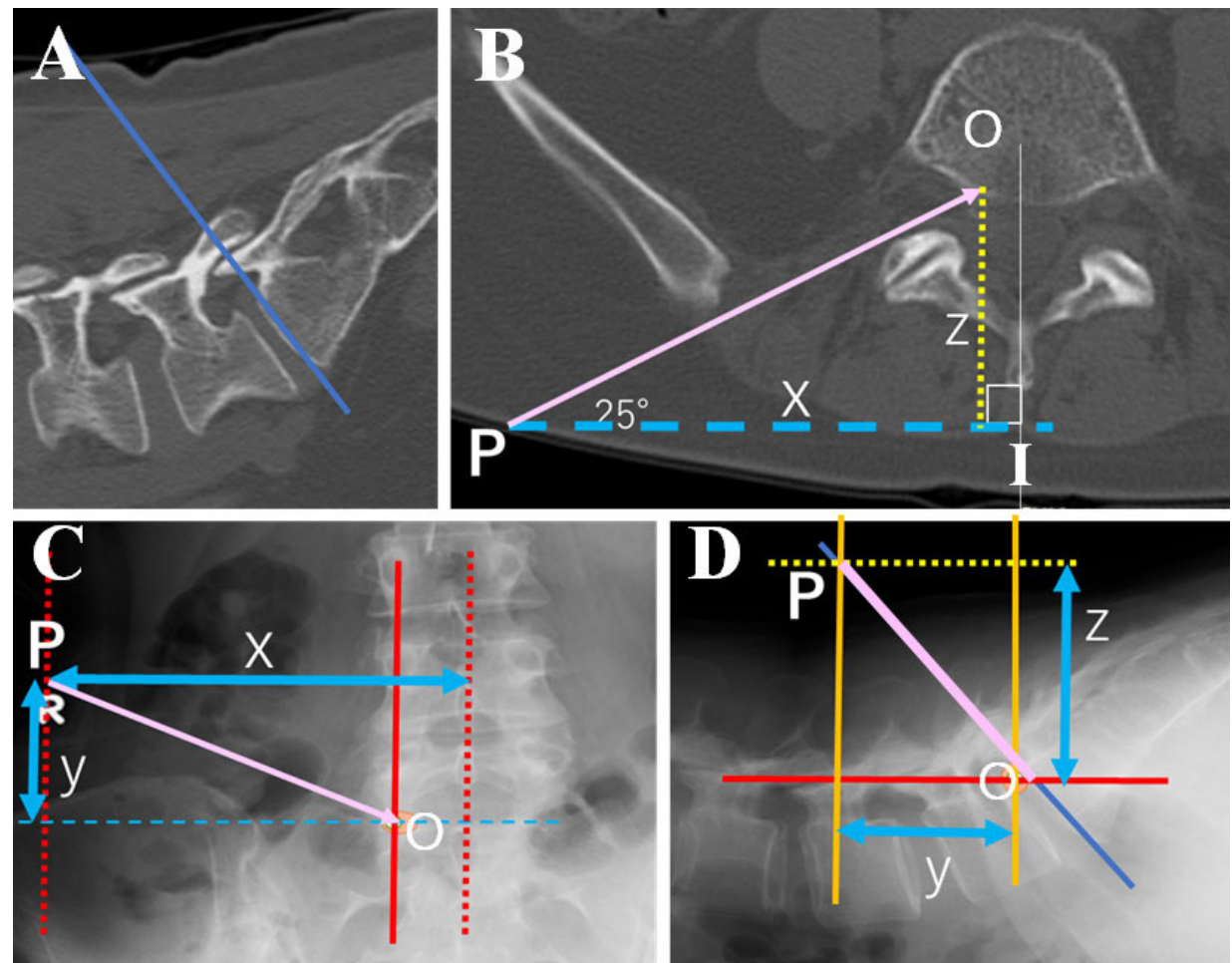

Figure 2: Preoperative design of entry points of skin (P) for L5/S1 lumbar disc herniation. (A) Axial computed tomography paralleled with target intervertebral disc (blue line) is scanned; (B) axial trajectory (pink line) is drawn with 25 degree angle against P-I line; (C, D) cranial (y) and dorsal $(\mathrm{z})$ deviations of $\mathrm{P}$ from the entry point of annulus fibrosus $(\mathrm{O})$ are also calculated
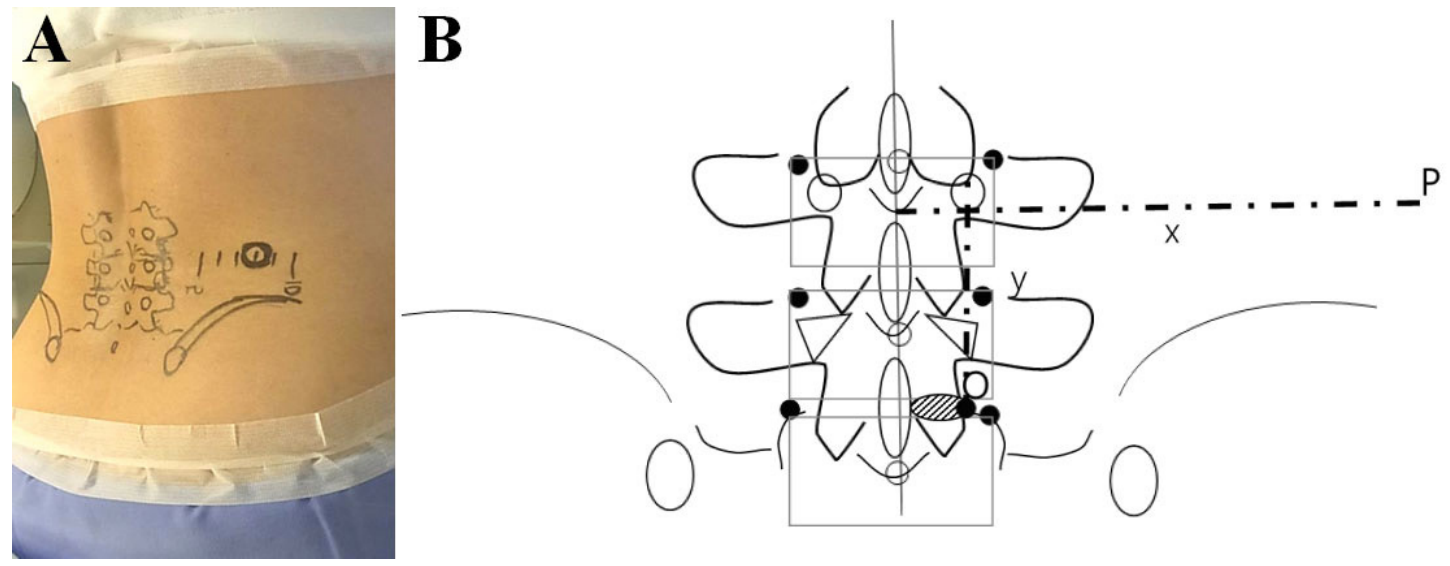

Figure 3: The entry points of skin $(\mathrm{P})$ and annulus fibrosus $(\mathrm{O})$ calculated by each distance $(\mathrm{x}, \mathrm{y}$, and $\mathrm{z})$ were draw together with anatomical bone structure (vertebral body, spinous process, transverse process, facet joint, and iliac crest) (A: intraoperative photography; B: enlarged schematic drawing) 

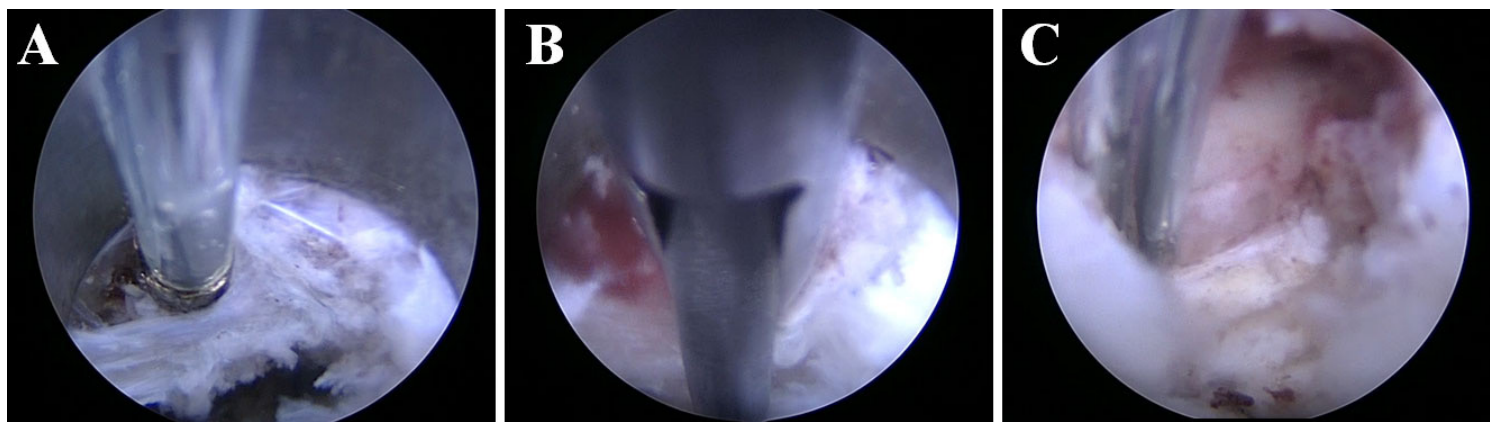

Figure 4: Upper half of the operative field is epidural space, lower half of that is disc spaces, and posterior longitudinal ligament (PLL) is consisting of the boundary of these spaces (A: PLL locates the tip of bipolar coagulator); (B) sharp dissection of the lateral margin of PLL is performed by beak forceps; (C) after that total removal of medial type lumbar disc herniation is achieved

dissection of the lateral part of PLL and subsequently electro-coagulation of the PLL [Figure 4B and C].

\section{Date analysis}

The operative outcomes were evaluated by two methods: (1) the change of pre- and postoperative numeric rating scale (NRS) scores of affected leg pain and NRS score of operative site pain; ${ }^{[11]}$ (2) MacNab's criteria rating activities of daily life at the most recent examination. According to the criteria, the results were described as excellent (completely pain free), good (minor intermittent discomfort, not interfering with normal activities), fair (improvement in symptoms but persistent backache or sciatica interfering with capacity to engage in full normal activities) and poor (no change in symptoms). ${ }^{[12]}$ Statistical analysis was performed with Student's paired $t$-test. $P$ values $<0.05$ were considered statistically significant.

\section{RESULTS}

A single level of PLPED for consecutive 48 cases was examined. The mean age of patients $(23$ men, 25 women) was 50.7 (range 17-88) years old. One patient underwent PLPED at L1-2 level, 3 at L2-3, 7 at L3-4, 29 at L4-5 and 8 at L5-S or L5-6 [Table 1].

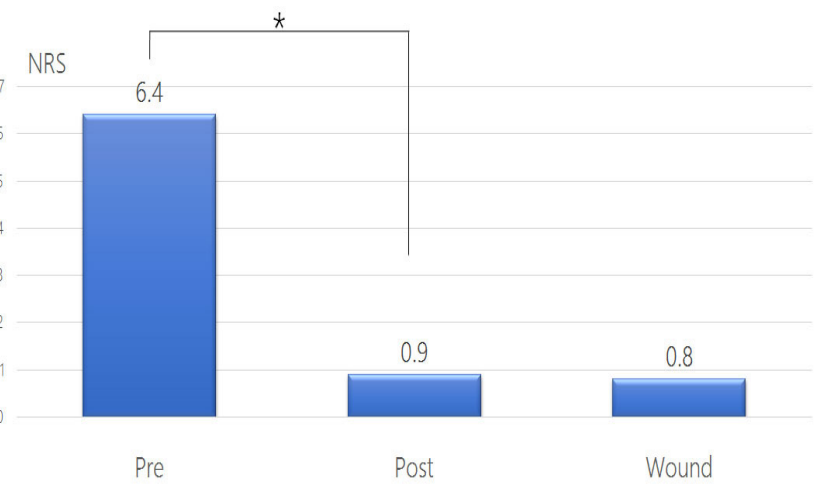

Figure 5: The short term operative outcome evaluated by numeric rating scale (NRS) score. Left and middle bar show the significant change of NRS score of affected leg pain $\left({ }^{*} P<0.05\right)$. Right bar is NRS score of operative site on the day following the operation
The short-term operative outcome was evaluated by NRS score. Post-operatively, NRS score of the affected leg significantly improved from 6.4 to $0.9(P<0.05)$ [Figure 5]. To evaluate wound pain, we also examined NRS score of operative site on the day following the operation. The mean NRS score was 0.8 in the next morning [Figure 5].

The long-term operative outcome was evaluated by MacNab's criteria. The patient was asked to rate the level of well-being at an average of 13.5 months after the operation (range: 1-30 months). "Excellent" or "good" ratings were obtained from $91.5 \%$ patients. "Fair" was obtained from $8.5 \%$ patients and no patient chose "poor" as per the MacNab's criteria [Table 2].

Mean operative time was 66.7 (range 38-152) min and intraoperative blood loss was negligible in all cases. Mean time to ambulation was 7.2 (range 2-20) $\mathrm{h}$. Mean hospital stay was 4.4 (range 1-33) days and the average duration of return to work was 17.2 (range 5-56) days [Table 3]. Although no serious complication occurred, 4 cases had minor transient neurological deficits. One patient $(2.1 \%)$ complained of dysesthesia of affected ENR area, but the dysesthesia has been gradually improving. Three patients $(6.3 \%)$ had a transient paresis that had completely disappeared after 3 months [Table 4]. No recurrence LDH was

Table 1: Background data of the patients

\begin{tabular}{lc}
\hline Characteristics & Data \\
\hline Gender & \\
Male & 23 \\
Female & 25 \\
Age (years), mean (range) & $50.7(17-88)$ \\
Level & \\
L1-2 & 1 \\
L2-3 & 3 \\
L3-4 & 7 \\
L4-5 & 29 \\
L5-S, L5-6 & 8 \\
Type of herniation & \\
Medial & 13 \\
Foraminal & 32 \\
Lateral & 3 \\
\hline
\end{tabular}


Table 2: The long term operative outcome evaluated by MacNab's criteria

\begin{tabular}{lc}
\hline MacNab's criteria & Data, $\boldsymbol{n}(\%)$ \\
\hline Excellent & $5(10.6)$ \\
Good & $39(80.9)$ \\
Fair & $4(8.5)$ \\
Poor & $0(0.0)$ \\
\hline
\end{tabular}

Table 3: The commencing times of walk and work

\begin{tabular}{lc}
\hline Characteristics & Time, mean (range) \\
\hline Start to walk & $7.2(2-20) \mathrm{h}$ \\
Hospital stay & $4.4(1-33)$ days \\
Return to work & $17.2(5-56)$ days \\
\hline
\end{tabular}

Table 4: Complication of posterolateral percutaneous endoscopic discectomy

\begin{tabular}{lc}
\hline Complications & Data, $\boldsymbol{n}(\%)$ \\
\hline Infection & $0(0)$ \\
Dysesthesia & $1(2.1)$ \\
Dural tear & $0(0)$ \\
Vascular injury & $0(0)$ \\
Transient palsy & $3(6.3)$ \\
Death & $0(0)$ \\
\hline
\end{tabular}

observed during the average 13.5 months follow-up (range 1-30).

\section{DISCUSSION}

As presented in this study, we describe the routine performance of detailed mapmaking for needle puncture of PLPED. This map includes entry points of skin $(P)$ and that of annulus fibrosus $(O)$ calculated by each distance ( $\mathrm{x}, \mathrm{y}$, and $\mathrm{z}$ ). Anatomical landmarks (spine, sacral ara, and iliac crest) are drawn together with these points. The map enables one to imagine underneath anatomical structures and to estimate obstruction of the puncture by iliac crest.

Accurate needle puncture of annulus fibrosus at the initial stage of PLPED requires significant experience, as inaccurate puncture may lead to the ENR injury. The position of entry points and the direction of the puncture is carefully designed by preoperative radiological images to achieve accurate and safe puncture. Especially for posterolateral approach to L5/ S1 LDH and/or high iliac crest, this map is essential. Even for the lateral type of L5/S1 LDH, which is a good indication for PLPED, high iliac crest disturbs removal of the medial part and the high grade migrated part of LDH. ${ }^{[13,14]}$ Sometimes partial facetectomy and outside-in technique is required for LDH combined with foraminal stenosis to prevent ENR injury. ${ }^{[15-19]}$ From our experience, these cases represented less than one third of the total cases and all successfully completed PLPED. The posterolateral approach is able to remove the migrated foraminal LDH except for the high grade upward migration at L5/S1 affected by iliac crest. ${ }^{[20]}$ The design strategy presented in this study utilizing preoperative images enables one to exclude the contraindicated cases. Those cases should be treated with the other posterior approaches, such as interlaminar and translaminar approaches. ${ }^{[20]}$

Discography and epidulography are very helpful to determine the trajectory of subsequent obturator insertion. Discography reveals the target disc space itself. Epidulography reveals the surface of the nucleus pulposus and the fragment and draws the Kambin's safety triangular zone closely located with ENR. These radiological intraoperative findings also support the preoperative mapping of the trajectory.

The complication rate of ENR injury in this study was $8.3 \%$ ( $n=4 / 48$ cases). This rate seems high, however most of the complications were transient neurological deficits and not prolonged. Even under general anesthesia, the majority of the patients could walk 2 h after surgery without lumbosacral orthosis. Furthermore, the long-term operative outcome as evaluated by MacNab's criteria, no patient chose the rating of "poor". Even under local anesthesia, ENR injury has been reported and the failure rates of percutaneous endoscopic lumbar discectomy range from $5 \%$ to $22 \%$. ${ }^{[21-24]}$

Free-running EMG monitoring has a potential to prevent ENR injury during percutaneous endoscopic lumbar discectomy. Although the EMG monitoring has been applied for to prevent motor deficits, the prevention of sensory deficits is lacking. Moreover, an exact value of free-running EMG monitoring has a diverse range amongst patients. In general, a threshold value of Neurovision ${ }^{\circledR}$ of $80 \mu \mathrm{V}$ is chosen. Depending on the patient's body habitus and the muscle mass, the threshold value may have to be changed (range: 10-300 $\mu \mathrm{V}$ ). One case demonstrating post-operative transient motor palsy was combined with foraminal stenosis of entry site (L5/S1). The obturator might compress the ENR at this site. No EMG changes were detected during the procedure, and the threshold of the EMG monitoring for this case should be decreased.

The end point of PLPED for the beginners is appearance of a pulsatile movement of ventral surface of dural sac just above the manipulated PLL. This situation shows at least a partial decompression that improves symptoms. Especially at the initial stage of this case series, several cases remained partial removal. However, a similar outcome was obtained even with cases compared to previously reported outcomes of total removal by microdiscectomy and microendoscopic discectomy. ${ }^{[25]}$

In conclusion, PLPED combining free-running EMG 
monitoring under general anesthesia is a safe and efficacious procedure for the treatment of LDH. The strict free-running EMG monitoring under general anesthesia makes both patients and surgeons comfortable and contributes to an improvement inoperative skill.

\section{DECLARATIONS}

\section{Authors' contributions}

Operated this series: Y. Kitahama

Suggested the idea of PED under general anesthesia: G. Matsui

Supported set-up of EMG monitoring: M. Minami, T. Kawaoka

Managed the general anesthesia of PED with EMG: K. Otome, M. Nakamura

\section{Financial support and sponsorship None.}

\section{Conflicts of interest \\ There are no conflicts of interest.}

\section{Patient consent \\ All involved patients gave their consent forms.}

\section{Ethics approval}

This study was approved by the ethics committee of the Omaezaki Municipal Hospital.

\section{REFERENCES}

1. Yeung AT, Yeung CA. Minimally invasive techniques for the management of lumbar disc herniation. Orthop Clin North Am 2007;38:363-72.

2. Fang G, Ding Z, Song Z. Comparison of the effects of epidural anesthesia and local anesthesia in lumbar transforaminal endoscopic surgery. Pain Physician 2016;19:E1001-4.

3. Kim KH. Use of lidocaine patch for percutaneous endoscopic lumbar discectomy. Korean J Pain 2011;24:74-80.

4. Weiss DS. Spinal cord and nerve root monitoring during surgical treatment of lumbar stenosis. Clin Orthop Relat Res 2001;(384):82-100.

5. Voulgaris S, Alexiou GA, Mihos E, Karagiorgiadis D, Zigouris A, Fotakopoulos G, Drosos D, Pahaturidis D. Posterior approach to ventrally located spinal meningiomas. Eur Spine J 2010;19:1195-9.

6. Holland NR. Intraoperative electromyography during thoracolumbar spinal surgery. Spine (Phila Pa 1976) 1998;23:1915-22.

7. Sairyo K, Kawamura T, Mase Y, Hada Y, Sakai T, Hasebe K, Dezawa A. Jack-knife stretching promotes flexibility of tight hamstrings after 4 weeks: a pilot study. Eur J Orthop Surg Traumatol 2013;23:657-63.

8. Sairyo K, Egawa H, Matsuura T, Takahashi M, Higashino K, Sakai T, Suzue N, Hamada D, Goto T, Takata Y, Nishisho T, Goda Y, Sato R, Tsutsui T, Tonogai I, Kondo K, Tezuka F, Mineta K, Sugiura K, Takeuchi M, Dezawa A. State of the art: transforaminal approach for percutaneous endoscopic lumbar discectomy under local anesthesia. J Med Invest 2014;61:217-25.

9. Ying J, Huang K, Zhu M, Zhou B, Wang Y, Chen B, Teng H. The effect and feasibility study of transforaminal percutaneous endoscopic lumbar discectomy via superior border of inferior pedicle approach for down-migrated intracanal disc herniations. Medicine (Baltimore) 2016;95:e2899.

10. Sairyo K, Higashino K, Yamashita K, Hayashi F, Wada K, Sakai T, Takata Y, Tezuka F, Morimoto M, Terai T, Chikawa T, Yonezu H, Nagamachi A, Fukui Y. A new concept of transforaminal ventral facetectomy including simultaneous decompression of foraminal and lateral recess stenosis: technical considerations in a fresh cadaver model and a literature review. J Med Invest 2017;64:1-6.

11. Schubert M, Hoogland T. Endoscopic transforaminal nucleotomy with foraminoplasty for lumbar disk herniation. Oper Orthop Traumatol 2005;17:641-61. (in English, German)

12. Macnab I. Negative disc exploration. An analysis of the causes of nerve-root involvement in sixty-eight patients. J Bone Joint Surg Am 1971;53:891-903

13. Yokosuka J, Oshima Y, Kaneko T, Takano Y, Inanam H, Koga H. Advantages and disadvantages of posterolateral approach for percutaneous endoscopic lumbar discectomy. J Spine Surg 2016;2:158-66.

14. Choi KC, Park CK. Percutaneous endoscopic lumbar discectomy for L5-S1 disc herniation: consideration of the relation between the iliac crest and L5-S1 disc. Pain Physician 2016;19:E301-8.

15. Li Z, Hou S, Shang W, Cao Z, Zhao H. Percutaneous lumbar foraminoplasty and percutaneous endoscopic lumbar decompression for lateral recess stenosis through transforaminal approach: technique notes and 2 years follow-up. Clin Neurol Neurosurg 2016;143:90-4.

16. Choi G, Lee SH, Lokhande P, Kong BJ, Shim CS, Jung B, Kim JS. Percutaneous endoscopic approach for highly migrated intracanal disc herniations by foraminoplastic technique using rigid working channel endoscope. Spine (Phila Pa 1976) 2008;33:E508-15.

17. Li ZZ, Hou SX, Shang WL, Song KR, Zhao HL. Modified percutaneous lumbar foraminoplasty and percutaneous endoscopic lumbar discectomy: instrument design, technique notes, and 5 years follow-up. Pain Physician 2017;20:E85-98.

18. Choi KC, Lee DC, Shim HK, Shin SH, Park CK. A strategy of percutaneous endoscopic lumbar discectomy for migrated disc herniation. World Neurosurg 2017;99:259-66.

19. Lee CW, Yoon KJ, Ha SS, Kang JK. Foraminoplastic superior vertebral notch approach with reamers in percutaneous endoscopic lumbar discectomy: technical note and clinical outcome in limited indications of percutaneous endoscopic lumbar discectomy. $J$ Korean Neurosurg Soc 2016;59:172-81.

20. Lee SH, Kang BU, Ahn Y, Choi G, Choi YG, Ahn KU, Shin SW Kang HY. Operative failure of percutaneous endoscopic lumbar discectomy: a radiologic analysis of 55 cases. Spine (Phila Pa 1976) 2006;31:E285-90.

21. Lee S, Kim SK, Lee SH, Kim WJ, Choi WC, Choi G, Shin SW. Percutaneous endoscopic lumbar discectomy for migrated disc herniation: classification of disc migration and surgical approaches. Eur Spine J 2007;16:431-7.

22. Du J, Tang X, Jing X, Li N, Wang Y, Zhang X. Outcomes of percutaneous endoscopic lumbar discectomy via a translaminar approach, especially for soft, highly down-migrated lumbar disc herniation. Int Orthop 2016;40:1247-52.

23. Schaffer JL, Kambin P. Percutaneous posterolateral lumbar discectomy and decompression with a 6.9-millimeter cannula. Analysis of operative failures and complications. J Bone Joint Surg Am 1991;73:822-31.

24. Wang H, Zhou Y, Li C, Liu J, Xiang L. Risk factors for failure of single-level percutaneous endoscopic lumbar discectomy. J Neurosurg Spine 2015;23:320-5.

25. Rasouli MR, Rahimi-Movaghar V, Shokraneh F, Moradi-Lakeh M, Chou R. Minimally invasive discectomy versus microdiscectomy/ open discectomy for symptomatic lumbar disc herniation. Cochrane Database Syst Rev 2014;(9):CD010328. 